\title{
Transcriptome analysis of 20 taxonomically related benzylisoquinoline alkaloid-producing plants
}

Jillian M. Hagel ${ }^{1}$, Jeremy S. Morris ${ }^{1}$, Eun-Jeong Lee ${ }^{1}$, Isabel Desgagné-Penix ${ }^{1,3}$, Crystal D. Bross $^{1}$, Limei Chang ${ }^{1}$, Xue Chen', Scott C. Farrow', Ye Zhang ${ }^{2}$, Jung Soh², Christoph W. Sensen ${ }^{2,4}$ and Peter J. Facchini ${ }^{1 *}$ (D)

\begin{abstract}
Background: Benzylisoquinoline alkaloids (BIAs) represent a diverse class of plant specialized metabolites sharing a common biosynthetic origin beginning with tyrosine. Many BIAs have potent pharmacological activities, and plants accumulating them boast long histories of use in traditional medicine and cultural practices. The decades-long focus on a select number of plant species as model systems has allowed near or full elucidation of major BIA pathways, including those of morphine, sanguinarine and berberine. However, this focus has created a dearth of knowledge surrounding non-model species, which also are known to accumulate a wide-range of BIAs but whose biosynthesis is thus far entirely unexplored. Further, these non-model species represent a rich source of catalyst diversity valuable to plant biochemists and emerging synthetic biology efforts.
\end{abstract}

Results: In order to access the genetic diversity of non-model plants accumulating BIAs, we selected 20 species representing 4 families within the Ranunculales. RNA extracted from each species was processed for analysis by both 1) Roche GS-FLX Titanium and 2) Illumina GA/HiSeq platforms, generating a total of 40 deep-sequencing transcriptome libraries. De novo assembly, annotation and subsequent full-length coding sequence (CDS) predictions indicated greater success for most species using the Illumina-based platform. Assembled data for each transcriptome were deposited into an established web-based BLAST portal (www.phytometasyn.ca) to allow public access. Homology-based mining of libraries using BIA-biosynthetic enzymes as queries yielded $\sim 850$ gene candidates potentially involved in alkaloid biosynthesis. Expression analysis of these candidates was performed using inter-library FPKM normalization methods. These expression data provide a basis for the rational selection of gene candidates, and suggest possible metabolic bottlenecks within BIA metabolism. Phylogenetic analysis was performed for each of 15 different enzyme/protein groupings, highlighting many novel genes with potential involvement in the formation of one or more alkaloid types, including morphinan, aporphine, and phthalideisoquinoline alkaloids. Transcriptome resources were used to design and execute a case study of candidate N-methyltransferases (NMTs) from Glaucium flavum, which revealed predicted and novel enzyme activities.

Conclusions: This study establishes an essential resource for the isolation and discovery of 1) functional homologues and 2) entirely novel catalysts within BIA metabolism. Functional analysis of G. flavum NMTs demonstrated the utility of this resource and underscored the importance of empirical determination of proposed enzymatic function. Publically accessible, fully annotated, BLAST-accessible transcriptomes were not previously available for most species included in this report, despite the rich repertoire of bioactive alkaloids found in these plants and their importance to traditional medicine. The results presented herein provide essential sequence information and inform experimental design for the continued elucidation of BIA metabolism.

\footnotetext{
* Correspondence: pfacchin@ucalgary.ca

'Department of Biological Sciences, University of Calgary, Calgary, AB T2N

1N4, Canada

Full list of author information is available at the end of the article
}

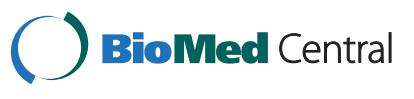

(c) 2015 Hagel et al. Open Access This article is distributed under the terms of the Creative Commons Attribution 4.0 International License (http://creativecommons.org/licenses/by/4.0/), which permits unrestricted use, distribution, and reproduction in any medium, provided you give appropriate credit to the original author(s) and the source, provide a link to the Creative Commons license, and indicate if changes were made. The Creative Commons Public Domain Dedication waiver (http://creativecommons.org/publicdomain/zero/1.0/) applies to the data made available in this article, unless otherwise stated. 


\section{Background}

Benzylisoquinoline alkaloids (BIAs) are a diverse class of plant specialized metabolites that includes approximately 2500 known compounds. Although BIAs present a wide range of structural backbone arrangements, they are united in their common biosynthetic origin, which begins with the condensation of two tyrosine derivatives forming the first dedicated BIA, (S)-norcoclaurine (Fig. 1). Several of humanity's most ancient medicines, poisons, hunting aids, and ceremonial preparations derive from plants accumulating BIAs, with examples found in both Old World and New World cultures [17]. Notable BIAaccumulating plants include morphine, codeine, and noscapine-accumulating opium poppy (Papaver somniferum), members of the berberine-accumulating barberry (Berberis) genus, Japanese goldthread (Coptis japonica), meadowrue (Thalictrum flavum), and species producing the antimicrobial sanguinarine, such as Mexican prickly poppy (Argemone mexicana) and California poppy (Eschscholzia californica). These plants form a core group of model species studied extensively in past decades, leading to the near-complete elucidation of major pathways at the biochemical and molecular genetic levels. Most or all enzymes responsible for the biosynthesis of papaverine, morphine, sanguinarine, berberine and noscapine have been cloned and characterized (Fig. 1) [6,17]. A restricted number of enzyme families have been implicated in BIA metabolism, which likely reflects a monophyletic origin for the pathway [34]. This feature has enabled homology-based enzyme discovery strategies, where predictions are made regarding enzyme type(s) acting at unresolved points along the BIA metabolic network. For example, $C-C$ or $C-O$ coupling reactions are almost exclusively catalyzed by cytochromes $\mathrm{P} 450$ with homology to one of CYP80, CYP82, or CYP719 families, or 2-oxoglutarate $/ \mathrm{Fe}^{2+}$-dependent dioxygenases. Resolution of previously uncharacterized steps in sanguinarine and noscapine metabolism has been achieved through homology-based querying of transcriptome resources coupled with targeted metabolite analysis [1,6,7]. This approach was used recently for the discovery of dihydrosanguinarine benzophenanthridine oxidase (DBOX), a FAD-dependent oxidase with homology to berberine bridge enzyme (BBE) [15]. Other enzyme types found repeatedly within BIA metabolism include $O$ - and $N$-methyltransferases, BAHD acylating enzymes [5] and reductases belonging to either aldo-keto (AKR) [39] or short-chain dehydrogenase/reductase (SDR) [23] superfamilies. Only the first step of BIA biosynthesis is catalyzed by a unique protein family, pathogenesis-related 10 (PR10)/Bet v1 allergens, otherwise absent within alkaloid metabolism (i.e. NCS; (S)-norcoclaurine synthase). Nonetheless, homologues of NCS appear to play this key entry-point role across different plant taxa [27].
Beyond model species, a myriad of other plants are known to accumulate BIAs. The structural diversity of these alkaloids is remarkable, yet their biosynthesis is largely or entirely unexplored. Many of these compounds have potent pharmacological activities, and plants accumulating them boast long histories of use in traditional medicine. Members of the Cissampelos genus, which accumulate novel bisbenzylisoquinoline, aporphine, and promorphinan-type alkaloids (Additional file 1) have been employed for centuries as hunting poisons and herbal remedies, particularly in South America and sub-Saharan Africa [45]. Trilobine, a highly crosslinked, atypical bisbenzylisoquinoline alkaloid, is thought to confer antiamoebic activity to herbal Cocculus preparations for the treatment of infant diarrhea [41]. Many plants of the Papaveraceae produce alkaloids featuring unique variations on the basic protoberberine and benzophenanthridine backbones, and some genus such as Corydalis accumulate a surprising variety of BIA types, including protopine, pthalideisoquinoline, spirobenzylisoquinoline, and morphinan alkaloids [21]. How these alkaloids are formed is poorly understood, and scarce resources are available for the non-model plants capable of producing them. To enable pathway elucidation and novel enzyme discovery, we have generated expansive datasets for twenty BIA-accumulating plants using Roche 454 and Illumina sequencing platforms. Data mining frameworks were constructed using a multitude of annotation approaches based on direct searches of public databases, and associated information was collected and summarized for every unigene, including Kyoto Encyclopedia of Genes and Genomes (KEGG) pathway maps, Gene Ontology (GO) and Enzyme Commission (EC) annotations. A comprehensive, broad-scope metabolite survey was performed in tandem with the herein presented transcriptome analysis, on identical plant tissues [18]. Used together, these unprecedented resources will allow the assembly of biochemical snapshots representing BIA metabolism in largely unexplored systems, guiding pathway elucidation and search efforts for new catalysts. Moreover, the availability of enzyme variants mined from different plant species will dramatically expand the 'toolbox' essential to synthetic biology efforts.

\section{Results and discussion}

\section{Species and tissue selection for enrichment of biosynthetic genes}

Twenty plant species were chosen for transcriptomic analysis, based primarily on alkaloid accumulation profiles, as determined by relevant literature sources and our concomitant study of metabolite content for candidate species [18]. Other considerations included taxonomic distribution, use in traditional medicine or cultural practices (signaling potential presence of pharmacologically 


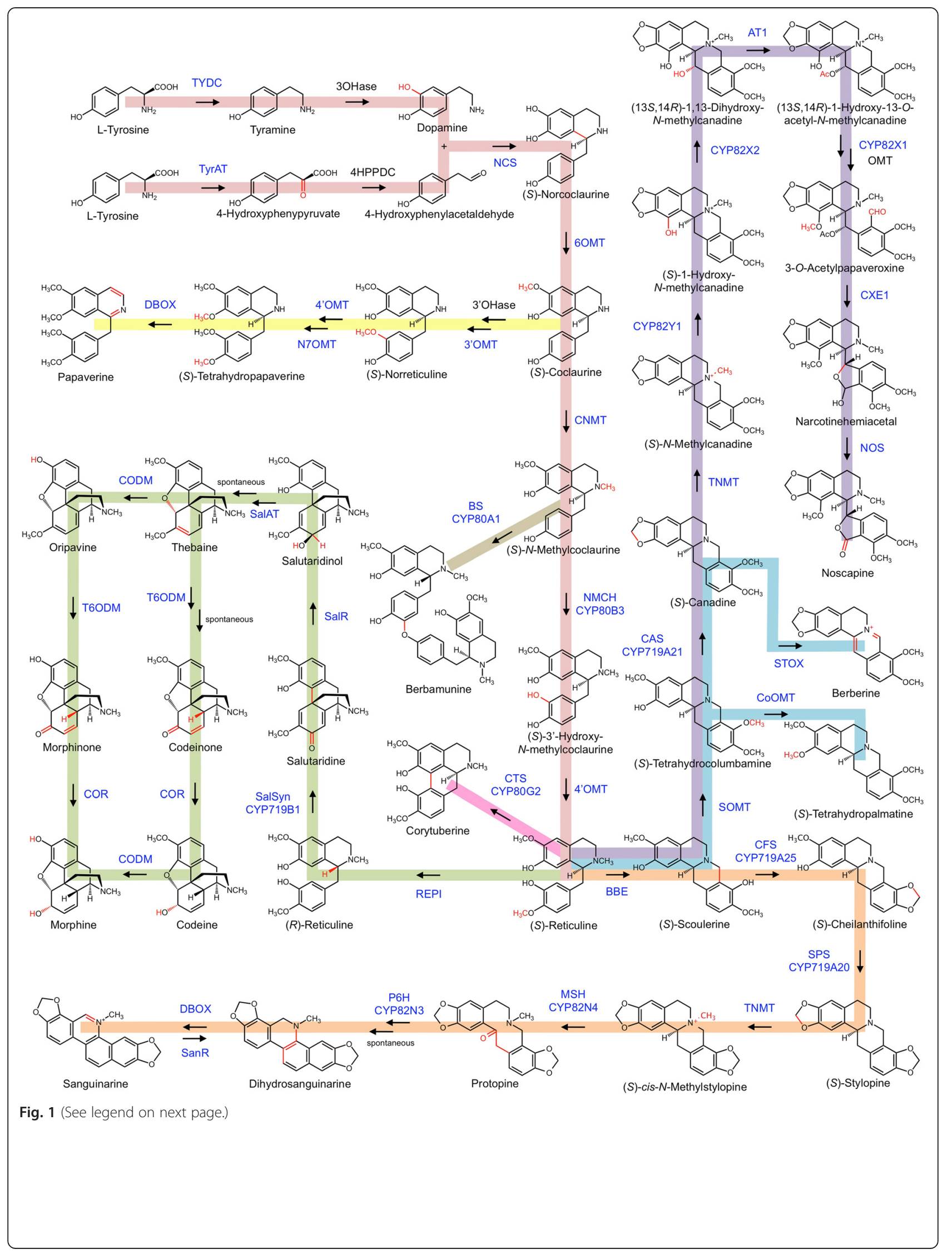


(See figure on previous page.)

Fig. 1 Major routes of BIA biosynthesis leading to (S)-reticuline (light pink), papaverine (yellow), morphine (green), sanguinarine (orange), berberine (blue) and noscapine (purple). C-O and C-C coupling reactions are shown for berbamunine (olive) and corytuberine (dark pink), respectively. Red within each alkaloid highlights enzyme-catalyzed structural changes. Solid and dotted arrows represent reactions catalyzed by single and multiple enzymes, respectively. Enzymes abbreviated in blue text have been characterized at the molecular level, whereas those in black text have not been cloned. Abbreviations: 3'-OHase, 3'-hydroxylase; 3'OMT, 3'-O-methyltransferase; 3OHase, 3-hydroxylase; 4HPPDC, 4-hydroxyphenylpyruvate decarboxylase; 4'OMT, 3'-hydroxy- $\mathrm{N}$-methylcoclaurine 4'-O-methyltransferase; 6OMT, norcoclaurine 6-O-methyltransferase; AT1, 1,13-dihydroxy- $\mathrm{N}$-methylcanadine 13-O-acetyltransferase; BBE, berberine bridge enzyme; BS, berbamunine synthase; CAS, canadine synthase; CFS, cheilanthifoline synthase; CNMT, coclaurine $\mathrm{N}$-methyltransferase; CODM, codeine O-demethylase; CoOMT, columbamine O-methyltransferase; COR, codeinone reductase; CTS, corytuberine synthase; CYP82X1, 1-hydroxy-13-O-acetyl-N-methylcanadine 8-hydroxylase; CYP82X2, 1-hydroxy-N-methylcanadine 13-hydroxylase; CYP82Y1, N-methylcanadine 1-hydroxylase; CDBOX, dihydrobenzophenanthridine oxidase; CXE1, 3-O-acetylpapaveroxine carboxylesterase; MSH, $\mathrm{N}$-methylstylopine hydroxylase; N7OMT, norreticuline 7-O-methyltransferase; NCS, norcoclaurine synthase; NMCanH, N-methylcanadine 1-hydroxylase; $\mathrm{NMCH}, \mathrm{N}$-methylcoclaurine 3'-hydroxylase; NOS, noscapine synthase; P6H, protopine 6-hydroxylase; REPI, reticuline epimerase; SalAT, salutaridinol 7-O-acetyltransferase; SalR, salutaridine reductase; SalSyn, salutaridine synthase; SanR, sanguinarine reductase; SOMT, scoulerine 9-O-methyltransferase; SPS, stylopine synthase; STOX, (S)-tetrahydroprotoberberine oxidase; T6ODM, thebaine 6-O-demethylase; TNMT, tetrahydroprotoberberine $\mathrm{N}$-methyltransferase; TYDC, tyrosine decarboxylase; TyrAT, tyrosine aminotransferase

active BIAs) and tissue availability. Priority was assigned to species for which sequence information was unavailable or lacking. We targeted four plant families within the order Ranunculales: the Papaveraceae (8 species), Ranunculaceae (4 species), Berberidaceae (4 species) and Menispermaceae (4 species) (Table 1). Although BIAs have been reported in diverse angiosperm taxa, they occur most commonly in these families [17]. Strong evidence supports the monophyletic origin of the Ranunculales, and within this order, the Papaveraceae family appears to have diverged early from the 'core' Ranunculales group (Additional file 2) [50]. Further evidence supports an early, monophyletic origin of BIA biosynthesis prior to the emergence of eudicots [34] suggesting that the last common ancestor of Ranunculales species was already making alkaloids. To enrich for BIA biosynthetic transcripts, analysis was restricted to alkaloid-rich organs (stem, rhizome, or root) or callus culture (Table 1). As an alternative to intact plants, cell cultures have been used for more than three decades as biosynthetic models and alkaloid production systems [54]. In vitro plant cell cultures have been instrumental in the discovery of several key enzymes and regulatory processes within sanguinarine, berberine, noscapine and morphine biosynthesis [17,44]. Recently, modest libraries ( 3500 unigenes) for 18 alkaloid-producing cultures, including callus of three Menispermaceae species, were established [10]. To build on these resources, callus of Cocculus trilobus, Tinospora cordifolia and Cissampelos mucronata were chosen for deep sequencing.

\section{Roche versus Illumina platforms: benefits of enhanced read depth}

RNA was screened for sufficient quality and quantity prior to deep sequencing by either Roche GS-FLX Titanium or Illumina GA/HiSeq platforms. For Illumina- based sequencing, GA (Genome Analyzer) and HiSeq instruments were employed to generate data of essentially equal quality, permitting subsequent pooling of the data. Table 2 summarizes the results for both technologies, while Additional files 3 and 4 tabulate further details regarding Roche and Illumina-based platforms, respectively. Data for 6 of the 20 species (Table 1) were published previously, although minor errors were noted (e.g. Table $1 \mathrm{~b}$ of [53]). Presented herein are corrected values, included for comparative purposes along with data for 14 new plant species. Multiplatform studies have highlighted certain advantages of Illumina-based sequencing over other technologies, which include lower costs $(\$ 0.06 / \mathrm{Mb})$, high accuracy $(<2 \%$ error rate) and good read depth, permitting robust transcript quantification $[32,40,46]$. Good read depth herein is reflected as high average reads per base pair (69.6; Additional file 4) permitting nearly double the number of average unigenes per library compared with Roche technology (34,368 versus 63,886, respectively; Table 2). Conversely, advantages of Roche 454 GS FLX-based sequencing include longer average read lengths (e.g. $>12$-fold longer than Illumina HiSeq platforms; [32]) enabling reliable detection of splice variants. Despite longer reads, Rochebased sequencing resulted in less predicted full-length coding sequences (CDSs) compared with Illuminabased sequencing (Additional files 3 and 4). Nonetheless, using two different platforms had the inherent advantage of enhanced overall transcriptome coverage. Roche and Illumina libraries averaged $\sim 14,000$ and $\sim 24,500$ full-length CDSs respectively, with an average of $\sim 7700$ CDS intersects between the libraries as determined by conservative, Mega BLAST estimates with an $e$-value cutoff of 0 ([56]; Additional file 3). The low number of CDS intersects likely reflects the use of stringent BLAST parameters rather than inherent differences between the two libraries, 
Table 1 Details of plant species selected for deep sequencing analysis

\begin{tabular}{|c|c|c|c|c|c|}
\hline \# & Species & Abbrev. & Common Name & Family (Tribe) & Organ/Tissue \\
\hline 1 & Argenome mexicana & AME & Mexican Prickly Poppy & Papaveraceae (Papaveroideae) & Stem \\
\hline 2 & Chelidonium majus & CMA & Greater Celandine & Papaveraceae (Papaveroideae) & Stem \\
\hline 3 & Papaver bracteatum & PBR & Persian Poppy & Papaveraceae (Papaveroideae) & Stem \\
\hline 4 & Stylophorum diphyllum & SDI & Celandine Poppy & Papaveraceae (Papaveroideae) & Stem \\
\hline 5 & Sanguinaria canadensis & SCA & Bloodroot & Papaveraceae (Papaveroideae) & Rhizome \\
\hline 6 & Eschscholzia californica & ECA & California Poppy & Papaveraceae (Papaveroideae) & Root \\
\hline 7 & Glaucium flavum & GFL & Yellow Horn Poppy & Papaveraceae (Papaveroideae) & Root \\
\hline 8 & Corydalis chelanthifolia & $\mathrm{CCH}$ & Ferny Fumewort & Papaveraceae (Fumarioideae) & Root \\
\hline 9 & Hydrastis canadensis & $\mathrm{HCA}$ & Goldenseal & Ranunculaceae & Rhizome \\
\hline 10 & Nigella sativa & NSA & Black Cumin & Ranunculaceae & Root \\
\hline 11 & Thalictrum flavum & TFL & Meadow Rue & Ranunculaceae & Root \\
\hline 12 & Xanthorhiza simplicissima & XSI & Yellowroot & Ranunculaceae & Root \\
\hline 13 & Mahonia aquifolium & MAQ & Oregon Grape & Berberidaceae & Bark \\
\hline 14 & Berberis thunbergii & BTH & Japanese Barberry & Berberidaceae & Root \\
\hline 15 & Jeffersonia diphylla & JDI & Rheumatism Root & Berberidaceae & Root \\
\hline 16 & Nandina domestica & NDO & Sacred Bamboo & Berberidaceae & Root \\
\hline 17 & Menispermum canadense & MCA & Canadian Moonseed & Menispermaceae & Rhizome \\
\hline 18 & Cocculus trilobus & CTR & Korean Moonseed & Menispermaceae & Callus \\
\hline 19 & Tinospora cordifolia & $\mathrm{TCO}$ & Heartleaf Moonseed & Menispermaceae & Callus \\
\hline 20 & Cissampelos mucronata & $\mathrm{CMU}$ & Abuta & Menispermaceae & Callus \\
\hline
\end{tabular}

and increasing the $e$-value cutoff would be expected to reveal greater concordance.

\section{Library comparisons reveal isolated cases of low intersection}

Variation in library quality between different source tissues (e.g. stem vs root, callus) was not apparent. For quality control measures, Illumina-based sequencing was performed on both stem and root of Chelidonium majus yielding comparable results (Additional file 5). However, library quality appeared reduced in isolated cases. For example, the Illumina-based Cocculus trilobus library consisted of a large number of reads, but yielded an above average number of unassembled contigs and a small number of full-length CDSs (Additional file 4). Conversely, Roche-based C. trilobus sequencing appeared relatively successful (Additional file 3). As Illumina- and Roche-based libraries were constructed using the same source material, we ruled out the possibility that $C$. trilobus tissue was compromised, as poor tissue quality would have affected both transcriptomes, not just the Illumina data. Another Illumina library with reduced full-length CDSs (compared to raw reads) and low intersection with Roche data included Mahonia aquifolium. It is possible that cross-contamination with samples derived from other plants occurred in these cases, precluding proper assembly and separation of foreign or native sequences at later stages.

\section{Establishment of fully annotated BLAST- accessible transcriptomes}

On average, $79 \%$ (Roche) and $69 \%$ (Illumina) of all unigenes received a functional annotation, with high-level annotations based on more stringent criteria assigned to $57 \%$ (Roche) and $50 \%$ (Illumina) (Table 2). Enzyme Commission (EC) number allocation was included in the analysis to gain insight on the number of enzymes represented in each library, and enable corresponding links to KEGG pathway maps (www.genome.jp/kegg/pathway). More importantly for enzyme discovery, EC assignments can facilitate word searches based on enzyme function. On average for both Roche and Illumina libraries, about $12 \%$ of all annotations corresponded to an EC number. Low success in EC number assignments was noted for C. trilobus and M. aquifolium Illumina libraries, likely due to poor assembly of full-length CDSs. Results for every unigene, including constituent reads, expression data, BLAST results, annotation evidence and relevant links are summarized on individual pages available through MAGPIE. A previously established MAGPIE- 
Table 2 Annotation summaries for Roche-based and Illumina-based transcriptomes

\begin{tabular}{|c|c|c|c|c|c|c|c|c|c|c|c|c|}
\hline \multirow[b]{2}{*}{ No. } & \multirow[b]{2}{*}{ Abbrev. } & \multirow[b]{2}{*}{ Plant } & \multicolumn{5}{|c|}{ Roche GS-FLX Titanium } & \multicolumn{5}{|c|}{ Illumina GA/HiSeq } \\
\hline & & & Unigenes & $\begin{array}{l}\text { Overall } \\
\text { annotated }\end{array}$ & $\begin{array}{l}\text { High-level } \\
\text { annotated }\end{array}$ & $\begin{array}{l}\text { GO } \\
\text { annotated }\end{array}$ & $\begin{array}{l}\text { EC number } \\
\text { allocated }\end{array}$ & Unigenes & $\begin{array}{l}\text { Overall } \\
\text { annotated }\end{array}$ & $\begin{array}{l}\text { High-level } \\
\text { annotated }\end{array}$ & $\begin{array}{l}\mathrm{GO} \\
\text { annotated }\end{array}$ & $\begin{array}{l}\text { EC number } \\
\text { allocated }\end{array}$ \\
\hline 1 & AME & $\begin{array}{l}\text { Argemone } \\
\text { mexicana }\end{array}$ & 25,499 & 22,121 & 17,979 & 21,974 & 3086 & 75,101 & 60,836 & 45,404 & 60,254 & 7653 \\
\hline 2 & BTH & $\begin{array}{l}\text { Berberis } \\
\text { thunbergii }\end{array}$ & 41,672 & 33,548 & 23,243 & 33,080 & 4197 & 88,302 & 61,576 & 41,927 & 60,561 & 7289 \\
\hline 3 & CMA & $\begin{array}{l}\text { Chelidonium } \\
\text { majus }\end{array}$ & 23,678 & 19,635 & 13,977 & 19,460 & 2368 & 45,005 & 42,057 & 33,449 & 41,956 & 6092 \\
\hline 4 & $\mathrm{CMU}$ & $\begin{array}{l}\text { Cissampelos } \\
\text { mucronata }\end{array}$ & 35,166 & 27,451 & 19,865 & 27,139 & 3147 & 69,822 & 32,209 & 22,943 & 31,597 & 3314 \\
\hline 5 & CTR & $\begin{array}{l}\text { Cocculus } \\
\text { trilobus }\end{array}$ & 34,783 & 26,678 & 18,701 & 26,338 & 3197 & 84,793 & 33,055 & 21,961 & 30,542 & 432 \\
\hline 6 & $\mathrm{CCH}$ & $\begin{array}{l}\text { Corydalis } \\
\text { chelanthifolia }\end{array}$ & 22,511 & 19,161 & 14,633 & 19,024 & 2433 & 51,797 & 48,423 & 42,784 & 48,139 & 7738 \\
\hline 7 & ECA & $\begin{array}{l}\text { Eschscholzia } \\
\text { californica }\end{array}$ & 32,150 & 28,430 & 21,403 & 28,194 & 4221 & 42,167 & 38,332 & 32,677 & 38,063 & 6545 \\
\hline 8 & GFL & $\begin{array}{l}\text { Glaucium } \\
\text { flavum }\end{array}$ & 26,520 & 20,945 & 15,645 & 20,725 & 2719 & 31,100 & 31,100 & 19,669 & 31,100 & 3231 \\
\hline 9 & HCA & $\begin{array}{l}\text { Hydrastis } \\
\text { canadensis }\end{array}$ & 23,809 & 20,443 & 15,491 & 20,230 & 2511 & 33,335 & 33,335 & 20,898 & 33,335 & 3637 \\
\hline 10 & JDI & $\begin{array}{l}\text { Jeffersonia } \\
\text { diphylla }\end{array}$ & 38,773 & 24,583 & 16,777 & 24,199 & 2581 & 86,832 & 31,712 & 22,574 & 30,842 & 3118 \\
\hline 11 & MAQ & $\begin{array}{l}\text { Mahonia } \\
\text { aquifolium }\end{array}$ & 36,429 & 30,209 & 20,624 & 29,805 & 3581 & 98,375 & 53,093 & 33,434 & 47,040 & 521 \\
\hline 12 & MCA & $\begin{array}{l}\text { Menispermum } \\
\text { canadense }\end{array}$ & 36,399 & 31,715 & 24,565 & 31,482 & 4495 & 87,141 & 70,524 & 52,713 & 69,877 & 8924 \\
\hline 13 & NDA & $\begin{array}{l}\text { Nandina } \\
\text { domestica }\end{array}$ & 45,387 & 33,501 & 24,308 & 33,010 & 4186 & 70,425 & 53,109 & 38,428 & 52,531 & 6553 \\
\hline 14 & NSA & Nigella sativa & 50,508 & 36,231 & 25,560 & 35,591 & 4526 & 67,591 & 41,260 & 29,127 & 40,316 & 4807 \\
\hline 15 & PBR & $\begin{array}{l}\text { Papaver } \\
\text { bracteatum }\end{array}$ & 46,224 & 33,168 & 24,381 & 32,767 & 4988 & 70,428 & 56,463 & 37,334 & 53,039 & 6793 \\
\hline 16 & SCA & $\begin{array}{l}\text { Sanguinaria } \\
\text { canadensis }\end{array}$ & 25,652 & 20,493 & 15,938 & 20,301 & 2621 & 53,019 & 47,247 & 40,122 & 46,890 & 7715 \\
\hline 17 & SDI & $\begin{array}{l}\text { Stylophorum } \\
\text { diphyllum }\end{array}$ & 43,568 & 34,954 & 26,144 & 34,614 & 5115 & 50,125 & 40,797 & 30,157 & 40,324 & 5276 \\
\hline 18 & TFL & $\begin{array}{l}\text { Thalictrum } \\
\text { flavum }\end{array}$ & 21,146 & 17,609 & 12,121 & 17,431 & 2294 & 41,982 & 33,120 & 23,900 & 32,711 & 4123 \\
\hline 19 & TCO & $\begin{array}{l}\text { Tinospora } \\
\text { cordifolia }\end{array}$ & 34,518 & 28,044 & 21,199 & 27,795 & 3444 & 81,927 & 35,851 & 24,174 & 34,712 & 3386 \\
\hline \multirow[t]{2}{*}{20} & XSI & $\begin{array}{l}\text { Xanthoriza } \\
\text { simplicissima }\end{array}$ & 42,969 & 33,657 & 22,165 & 33,187 & 3740 & 48,447 & 39,281 & 27,434 & 38,831 & 4642 \\
\hline & & Average & 34,368 & 27,128 & 19,736 & 26,817 & 3472 & 63,886 & 44,169 & 32,055 & 43,133 & 5089 \\
\hline
\end{tabular}

based BLAST portal [53] is available for public access to the assembled data of each transcriptome reported herein (www.phytometasyn.ca).

\section{Homology-based mining of BIA biosynthetic genes}

Illumina and Roche 454-based transcriptomes were mined for candidate genes putatively involved in BIA metabolism. tBLASTn searches were performed on the basis of homology to fully characterized alkaloid biosynthetic enzymes, using a cutoff of $40 \%$ sequence identity in most cases. Exceptions include O-acetyltransferases (OATs) and carboxylesterases (CXEs) where a search cutoff of $30 \%$ was generally used. For OATs and CXEs, greater sequence divergence between taxonomic groups was evident, prompting more flexible search criteria. A pre-defined cutoff was not required in some cases, since 
tBLASTn yielded a small number of hits with relatively high identity. For example, searches using berberine bridge enzyme from Eschscholtzia californica, Papaver somniferum and Berberis stolonifera (EsBBE, PsBBE and BsBBE respectively) yielded a total of 18 hits with substantial $(>60 \%)$ identity. Similar results were obtained for dihydrobenzophenanthridine oxidase (DBOX)-like FAD-dependent oxidases (FADOX). In total, $\sim 850$ candidate unigenes were selected from 40 deep sequencing libraries, representing 20 BIA-accumulating plant species. Additional file 6 lists the amino acid sequences of these candidates in FASTA format.

\section{Gene expression for candidate selection and bottleneck identification}

Expression data were recorded for each candidate in the form of FPKM (Fragments Per Kilobase of exon model per Million mapped reads) extracted from Illumina libraries. Figure 2 summarizes results obtained for Papaveroideae tribe members (Papaveraceae). Expression results for Corydalis chelanthifolia (Fumarioideae tribe, Papaveraceae), Berberidaceae and Ranunculaceae species are found in Additional file 7, and results for Menispermaceae species are found in Additional file 8. Expression analyses were not performed for $M$. aquifolium and C. trilobus due to reduced numbers of full-length CDSs. Expression values were normalized across all Illumina libraries, permitting cross-species comparison (see methods). FPKM and related RNA-seq tools are reliable expression metrics; in fact, recent head-tohead comparison of Illumina and microarray-based data showed that RNA-seq dramatically outperforms microarray in identifying differentially expressed genes [49]. For the purpose of novel catalyst discovery, gene expression data can be used to prioritize candidates for further analysis. Genes highly expressed in BIAsynthesizing tissues can be selected over candidates with very low expression levels. For example, while $17 \mathrm{pu}$ tative (S)-norcoclaurine synthase (NCS) candidates were identified within Papaveraceae libraries, some of these unigenes were observed only as low-read Roche contigs and were entirely absent from Illumina data (Fig. 2, Additional file 7). Lack of Illumina data could reflect a platform bias or processing error, although it is possibly the result of very low gene expression. Expression comparisons can be made across different gene families to gain insight regarding putative metabolic bottlenecks. Papaver bracteatum accumulates large quantities of thebaine but only trace amounts of downstream alkaloids codeine and oripavine [24], implicating a metabolic block at thebaine 6-O-demethylase (T6ODM) and codeine O-demethylase (CODM) (Fig. 1). T6ODM and CODM have been characterized in opium poppy and belong to the $\mathrm{Fe}^{2+} / 2$-oxoglutaratedependent dioxygenase (DIOX) family [16]. Compared with other BIA-biosynthetic genes in P. bracteatum, DIOX homologues are expressed at very low levels, possibly contributing to observed pathway restrictions.

\section{Phylogenetic analysis as prediction tool for gene function: NMT case study}

Amino-acid alignments and phylogenetic trees were assembled for 15 classes of protein/enzymes, representing a total of $\sim 850$ gene candidates. Figures 3 and 4 illustrate the trees built using CYP719 and $N$-methyltransferase candidates, respectively. Remaining trees are found in the Additional files 9, 10, 11, 12, 13, 14, 15, 16, 17, 18, 19, 20 and 21. Used together with the corresponding FPKM data and species-specific alkaloid profiles [18] these results represent an important resource for the discovery of new enzymes catalyzing (i) previously characterized reactions (i.e. functional homologues) and (ii) reactions uncharacterized at the biochemical and molecular levels. To test our hypothesis that phylogenetic considerations could be used to predict enzyme function, we designed an empirical case study using Glaucium flavum N-methyltransferase (NMT) gene candidates. Homology-based mining revealed six full-length NMT candidates in both Roche- and Illumina-based G. flavum transcriptomes (Fig. 2). Phylogenetic analysis revealed closer relationships between certain G. flavum candidates to characterized enzymes compared to others. For example, GFLNMT1 formed a six-member clade with PSOCNMT, an established coclaurine $\mathrm{N}$ methyltransferase (CNMT) from Papaver somniferum [19] (Fig. 4). In contrast, GFLNMT2 formed a 6member clade including (S)-tetrahydroprotoberberine $\mathrm{N}$-methyltransferase (TNMT) from Eschscholzia californica (ECATNMT) [35]. On the basis of these results, it was predicted that GFLNMT1 and GFLNMT2 enzymes would exhibit CNMT and TNMT activities, respectively. Although the remaining GFLNMTs did not form similarly small clades with, or exhibit such high identity $(>70 \%)$ to known enzymes, activity with BIA substrates was anticipated owing to the $>40 \%$ identity with query sequences. All six G. flavum candidates were produced in Escherichia coli as His-tagged recombinant proteins, each of which showed a predicted molecular weight as determined by comparison with molecular weight standards (Additional file 22). Each protein was tested for NMT activity using six key alkaloid substrates (Table 3). Indeed, GFLNMT1 and GFLNMT2 exhibited CNMT and TNMT activities using coclaurine and protoberberine substrates, respectively. Further, our prediction that all G. flavum enzymes would accept BIA substrates proved correct. GFLNMT3 acted as TNMT using $(S)$-stylopine substrate, but unexpectedly also $N$-methylated $(S)$ reticuline. (S)-Reticuline $N$-methyltransferase activity was also observed for GFLNMT5. GFLNMT4 acted as CNMT 


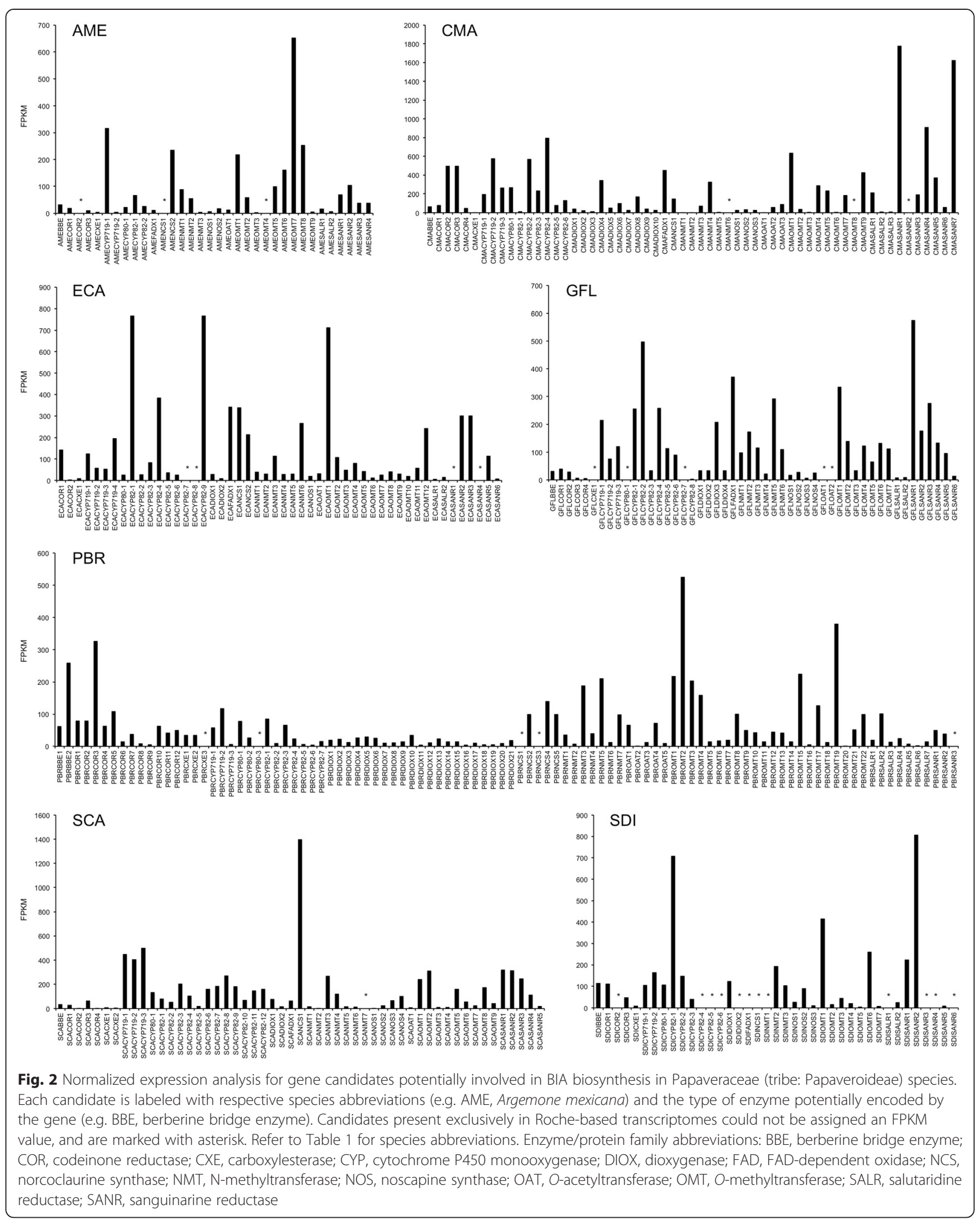




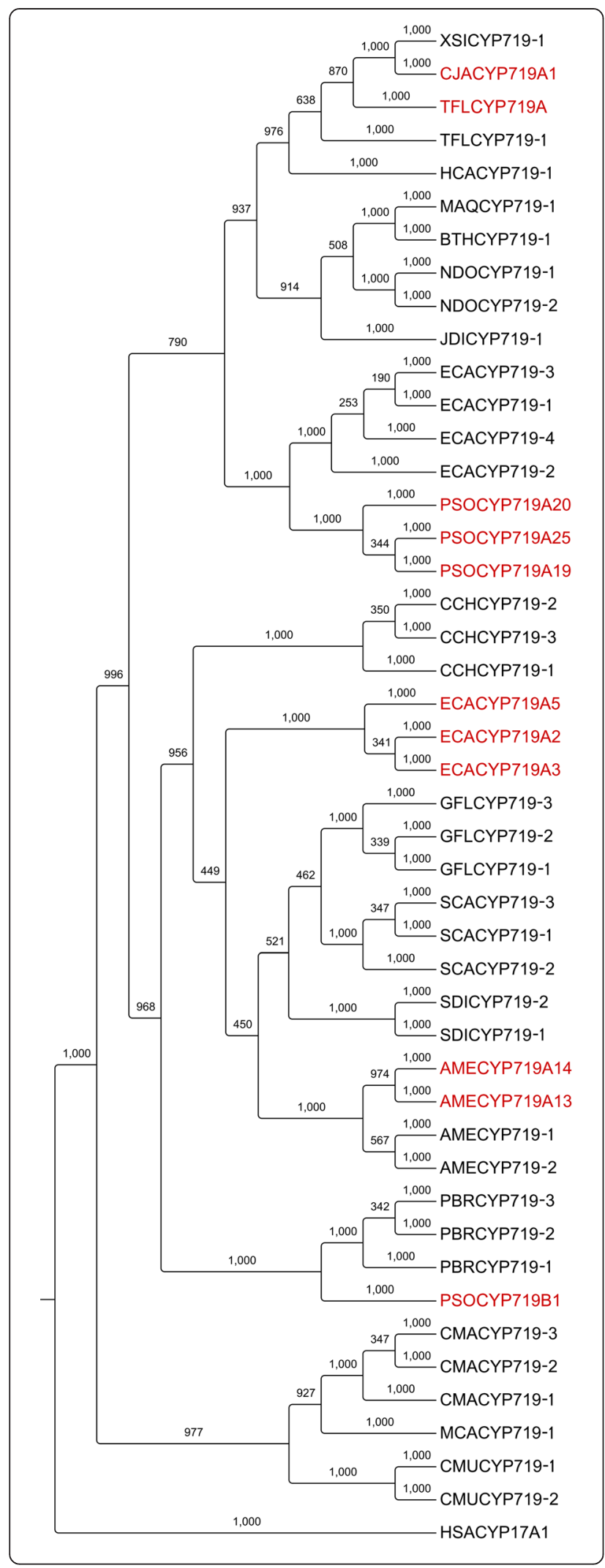

Fig. 3 Phylogenetic analysis of CYP719 gene candidates from twenty BIA-accumulating plant species. Red text denotes characterized genes or enzymes used as tBLASTn queries for transcriptome mining. Black text denotes uncharacterized gene candidates identified through mining (>40 \% identity to queries). Bootstrap values for each clade were based on 1000 iterations. Each candidate is labeled with respective species abbreviation (e.g. AME, Argemone mexicana; see Table 1) and candidate number (e.g. CYP719-1). Each query is labeled according to species (additional species: CJA, Coptis japonica; PSO, Papaver somniferum) with CYP719 subfamily and gene number indicated (e.g. CYP719B1, salutaridine synthase; see Fig. 1). Outgroup is CYP17A1 from Homo sapiens (HSA). Amino acid sequences for candidates, queries, and outgroups are found in Additional file 6

with the notable distinction of carrying out subsequent $N, N$-dimethylation reactions to form a quaternary amine. Although GFLNMT6 did not cluster closely with characterized CNMT (Fig. 4), it accepted coclaurine substrate. These results demonstrate the general utility of phylogenetic analysis as a predictive tool, but underscore the need for empirical assay data for the purposes of gene discovery.

\section{Functional homologue resource for synthetic biology}

For the purposes of emerging synthetic biology initiatives, functional homologues - often termed enzyme 'variants' are essential engineering tools. Assembly of alkaloid pathways in microbes using heterologously expressed plant enzymes is fraught with problems - including poor protein expression, unpredictable/off-target activities, poor interaction with other pathway enzymes, and low catalytic efficiencies [28] - which can be alleviated in some cases with variant substitution. For example, testing numerous combinations of methyltransferases from Papaver somniferum and Thalictrum flavum revealed that specific variants, and combinations of variants, ameliorated $(S)$-reticuline production in yeast [19]. Our collection of $N$ - and $O$-methyltransferase candidates sourced from a wide variety of plants (Fig. 4, Additional file 18) will enable further refinement of alkaloid biosynthesis in unicellular systems.

\section{Candidates with putative roles in morphinan and aporphine alkaloid formation}

Identification of functional homologues with roles in morphinan alkaloid biosynthesis is an important objective, as reconstitution of this pathway in microbes is an emerging goal [48]. The Illumina transcriptome of morphinan alkaloid-producing $P$. bracteatum contains three CYP719 candidates, which form a well-supported clade with opium poppy (Papaver somniferum) salutaridine synthase (SalSyn, PSOC719B1; Fig. 3). In addition, six P. bracteatum unigenes with substantial homology (up to $92 \%$ amino acid identity) to opium poppy salutaridine 


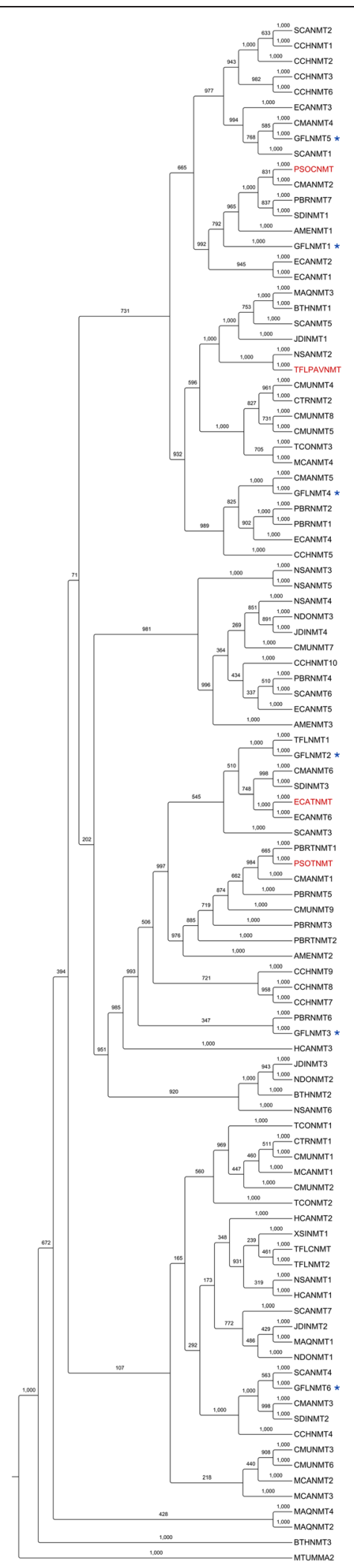

Fig. 4 Phylogenetic analysis of $\mathrm{N}$-methyltransferase (NMT) gene candidates from twenty BIA-accumulating plant species. Red text denotes characterized genes or enzymes used as tBLASTn queries for transcriptome mining. Black text denotes uncharacterized gene candidates identified through mining ( $>40 \%$ identity to queries). Bootstrap values for each clade were based on 1000 iterations. Each candidate is labeled with respective species abbreviation (e.g. AME, Argemone mexicana; see Table 1) and candidate number (e.g. NMT1). Each query is labeled according to species (additional species: PSO, Papaver somniferum) and specific NMT function (CNMT, coclaurine $\mathrm{N}$-methyltransferase; PAVNMT, pavine $\mathrm{N}$-methyltransferase; TNMT, tetrahydroprotoberberine N-methyltransferase; see Fig. 1). Outgroup is mycolic acid synthase from Mycobacterium tuberculosis (MTUMMA2). NMT candidates from Glaucium flavum tested for catalytic activity are indicated with asterisks. Amino acid sequences for candidates, queries, and outgroups are found in Additional file 6

reductase (SalR) were identified (Fig. 2, Additional file 14). Our study includes plant genera known to produce lesserknown morphinan alkaloids, such as Corydalis, Nandina and Thalictrum, which produce (+)-pallidine, sinoacutine, and $(-)$-pallidine respectively $[21,22,47]$. Significantly, these plants also produce a variety of aporphine alkaloids such as nantenine (Nandina; [22]), isocorydine (Corydalis; [14]) and corydine (Thalictrum; [47]). The biosynthetic pathways for these morphinan and aporphine alkaloids are not known, but likely rely on CYP-mediated $C$-C coupling of $(S)$ - or $(R)$-reticuline. The relatively few $(<10)$ CYP80, CYP719 and CYP82 candidates were identified in these species (Fig. 3, Additional files 16 and 17) could be tested for reticuline oxidase activity and evaluated for participation in morphinan and/or aporphine pathways.

\section{Potential new catalysts for phthalideisoquinoline alkaloid biosynthesis}

Guided by the recent elucidation of noscapine biosynthesis in opium poppy [6,51], transcriptomes of phthalideisoquinoline-accumulating species were mined for novel catalysts. Hydrastis canadensis produces hydrastine, hydrastidine, and other minor constituents [26] whereas Corydalis species accumulate a wide variety of phthalideisoquinoline alkaloids [2]. Numerous acetyltransferase, carboxylesterase, and CYP82 candidates with possible involvement in phthalideisoquinoline biosynthesis were identified in $H$. canadensis and C. chelanthifolia transcriptomes. Corydalis species accumulate the hemiacetal egenine [52], which may require a noscapine synthase (NOS)-like enzyme for hypothesized conversion to bicuculline [12]. Six candidates were identified in C. chelanthifolia with up to $52 \%$ identity to $P$. somniferum NOS, although expression was very low in some cases (Additional file 7). Three NOS-like gene candidates with possible roles as hydrastine synthases were identified in H. canadensis (Additional files 7 and 21). 
Table 3 Relative conversion of five alkaloids tested as potential substrates for six NMT candidates from Glaucium flavum

\begin{tabular}{|c|c|c|c|c|c|c|}
\hline \multirow[t]{2}{*}{ Substrate } & \multicolumn{6}{|c|}{ Relative activity (\% maximum) } \\
\hline & GFLNMT1 & GFLNMT2 & GFLNMT3 & GFLNMT4 & GFLNMT5 & GFLNMT6 \\
\hline (S)-Coclaurine & $100 \pm 1^{\mathrm{a}}$ & nd & nd & $100 \pm 10^{d, e}$ & $<1$ & $100 \pm 14^{9}$ \\
\hline (S)-Reticuline & nd & nd & $100 \pm 5^{c}$ & $8 \pm 1$ & $100 \pm 5^{f}$ & nd \\
\hline (S)-Canadine & nd & $66 \pm 1$ & $<1$ & nd & nd & nd \\
\hline (S)-Stylopine & nd & $100 \pm 2^{b}$ & $81 \pm 18$ & nd & nd & nd \\
\hline (+/-)-Pavine & nd & $<1$ & nd & nd & nd & nd \\
\hline
\end{tabular}

Values represent the mean \pm standard deviation of three independent assays. For each enzyme, activity was calculated relative to the assay showing the highest conversion of substrate (i.e. the average of this assay was set to $100 \%$ ). The accompanying footnote defines $100 \%$ conversion in pmole min ${ }^{-1} \mathrm{mg}^{-1} \mathrm{protein}_{\text {for }}$ each enzyme

${ }^{\mathrm{a}} 5.9$ pmole $\mathrm{min}^{-1} \mathrm{mg}^{-1}$ protein

${ }^{\mathrm{b}} 61$ pmole $\mathrm{min}^{-1} \mathrm{mg}^{-1}$ protein

0.4 pmole $\mathrm{min}^{-1} \mathrm{mg}^{-1}$ protein

${ }^{d}$ Products were $\mathrm{N}$-methylcoclaurine and $\mathrm{N}, \mathrm{N}$-dimethylcoclaurine

e 2.3 pmole $\mathrm{min}^{-1} \mathrm{mg}^{-1}$ protein

f 0.1 pmole $\mathrm{min}^{-1} \mathrm{mg}^{-1}$ protein

${ }^{9} 3.3$ pmole $\mathrm{min}^{-1} \mathrm{mg}^{-1}$

\section{Conclusions}

The establishment of fully annotated, deep-sequencing transcriptomes for twenty BIA-accumulating plants represents an immense resource for novel catalyst discovery. BLAST-accessible transcriptomes were not previously available for most plants included in this report, despite the rich repertoire of bioactive alkaloids found in these species and their importance in traditional medicine. The results presented herein, together with accompanying metabolite profiles [18] and relevant literature, are intended to provide necessary tools (i.e. gene sequences) and also inform experimental design for the continued elucidation of the BIA metabolism.

\section{Methods \\ Alkaloids}

Alkaloids used as substrates or standards were sourced as follows: $(S)$-reticuline oxalate was a gift from Tasmanian Alkaloids (Tasmania, Australia); $(R, S)$-canadine was purchased from Latoxan (Valence, France); $( \pm$ )-pavine was purchased from Sigma-Aldrich (St. Louis, MO), (S)coclaurine was purchased from Toronto Research Chemicals (Toronto, ON); $(R, S)$-stylopine was synthesized as described previously [33].

\section{Plant material}

Selected tissues were harvested from Hydrastis canadensis, Sanguinaria canadensis, Nigella sativa, Mahonia aquifolium, Menispermum canadense, Stylophorum diphyllum, and Xanthoriza simplicissima plants cultivated outdoors at the Jardin Botanique de Montréal (Montréal, Québec; http://espacepourlavie.ca). Jeffersonia diphylla and Berberis thunbergii plants were purchased from Plant Delights Nursery (Raleigh, North Carolina; www.plantdelights.com) and Sunnyside Greenhouses (Calgary, Alberta; www.sunnysidehomeandgarden.com), respectively. Chelidonium majus, Papaver bracteatum, Argemone mexicana, Eschscholtzia californica, Nandina domestica, Glaucium flavum, Thalictrum flavum and Corydalis chelanthifolia were grown from seed germinated in potted soil under standard open air greenhouse conditions at the University of Calgary (Calgary, Alberta). Seeds were obtained from B and T World Seeds (b-and-t-world-seeds.com) with the exception of $T$. flavum and P. bracteatum, which were obtained from Jelitto Staudensamen (www.jelitto.com) and La Vie en Rose Gardens (www.lavieenrosegardens.com), respectively. Callus cultures of Cissampelos mucronata, Cocculus trilobus, and Tinospora cordifolia were purchased from Deutsche Sammlung von Mikroorganismen und Zellkulturen (DSMZ, Braunschweig, Germany; www.dsmz.de) and maintained as described [10]. All tissues were flash-frozen in liquid nitrogen and stored at $-80{ }^{\circ} \mathrm{C}$ until analysis.

\section{Poly(A) + RNA purification, cDNA library preparation and next-generation sequencing}

Total RNA was extracted from stem, rhizome, root, or callus tissue using a modified CTAB method [38]. RNA quality was based on UV absorption ratios, where only samples with ratios above $2.0(260 / 280 \mathrm{~nm})$ and 2.2 $(260 / 230 \mathrm{~nm})$ were used. Poly(A) + RNA purification, cDNA library synthesis, emulsion-based PCR (emPCR) and NGS was performed at the McGill University and Génome Québec Innovation Center (Montréal, Québec) as described [53]. Briefly, RNA quality and quantity was assessed using NanoDrop ND-1000 (Thermo Scientific, Waltham, Massachusetts) and BioAnalyzer 2100 (Agilent Technologies, Santa Clara, California) instruments, and Poly(A) + RNA purification was done using either a Dynabeads mRNA Purification kit (Invitrogen) or TrueSeq Stranded mRNA Sample Prep kit (Illumina, San Diego, California). cDNA synthesis was performed 
using either a cDNA Rapid Library kit (Roche, Basel, Switzerland) or TruSeq Stranded mRNA Sample Prep kit (Illumina) depending on the downstream NGS method. For Roche-454 GS-FLX Titanium pyrosequencing, data processing was done using GS Run Processor (Roche) to generate Standard Flowgram Format (SFF) files. For Illumina GA and HiSeq sequencing, HCS 1.4 and CASAVA 1.6-1.8 software suites (Illumina) were used to generate raw fastq reads.

\section{De novo transcriptome assembly, functional annotation, and $\mathrm{GO}$ analysis}

Sequence quality control and screening was performed as described [53]. Adapter/primer sequences were clipped, all sequences were trimmed based on Phred quality scores, low-complexity regions were masked, and ribosomal RNA (rRNA) sequences were removed from each 454 database using the Scylla program of the Paracel Filtering Package (PFP) (Paracel Inc., California). Quality assessment and cleaning for Illumina reads was performed using Fast QC (www.bioinformatics.babraham.ac.uk/projects/ fastqc/) and Cutadapt [37]. Cleaned 454 sequence data were assembled using MIRA (v. 3.2) [4], which produced more long (>1000 bp) contigs compared with Paracel Transcript Assembler (Paracel Inc.) or Newbler v. 2.3 [36] platforms. Filtered Illumina reads were assembled using Velvet-Oases v. 0.1.16 [55]. CD-HIT-EST [30] was used to reduce redundancy by clustering of nearly identical ( $>99 \%)$ transcripts, and further assembly was achieved using CAP3 [20]. MAGPIE (Magpie Automated Genomics Project Investigation Environment) [11] was used to annotate each dataset based on sequence similarity searches against public and internal databases, including NCBI and the viridiplantae subset of RefSeq. Accelerated Hidden Markov Model (HMM) searches were also performed. Full-length coding sequence predictions were performed as described [53]. Functional descriptions based on comparison with already annotated sequences from databanks, along with domain-level contents, were assigned to each contig based on a weighted summary of all the search results. GO (Gene Ontology) annotations and EC numbers were designated for each contig as described previously [53]. Toward the goal of integrating transcriptomic with corresponding metabolomic data [18] transcript data was mapped to KEGG metabolic pathways using EC numbers.

\section{Gene expression analysis}

As a first round, relative gene expression information for every contig in all 40 libraries (twenty 454-based, and twenty Illumina-based) was acquired based on raw read abundance. For 454 libraries, raw read counts were extracted from contig assembly files. For Illumina libraries, counts were estimated using Bowtie [25] to re-map raw reads to assembled contigs, and RSEM [29] was used for final quantifications. Relative normalization (i.e. within each respective library) was achieved by calculating FPKM (Fragments Per Kilobase of exon model per Million mapped reads) for each contig. To enable gene expression comparisons across different libraries, a second round of normalization was performed using Illumina data. First, contigs from all twenty Illumina libraries were compiled together and grouped into clusters based on sequence similarity. Clustering of data was performed using OrthoMCL, a program designed for the scalable construction of orthologous groups across multiple eukaryotic taxa [31]. Differences in RNA quantity between libraries (i.e. RNA composition bias) were accounted for by calculating a combined scaling factor for each library. This step was performed using the calcNormFactors function of edgeR v.3 (www.bioconductor.org), which determines a set of factors, later combined into a single "scaling factor", unique to each library that minimizes log-fold changes between samples for most genes [3]. A second set of FPKM values enabling crossspecies comparison were generated for contigs of interest through multiplication of the first, library-specific FPKM values by respective scaling factors.

\begin{abstract}
Alignments and phylogenetic analysis of gene candidates Amino acid alignments of candidates belonging to individual enzyme classes were performed with the in-built Muscle alignment feature of Geneious (Biomatters, Aukland, New Zealand). The alignments were performed as a free-end gap, and computational alignments were followed by hand sorting. Maximum-likelihood phylogenetic analyses were performed using the PHYML feature of Geneious [13]. Bootstrap values for each clade were based on 1000 iterations. For P450 and NMT trees, Homo sapiens and bacterial (Mycobacterium tuberculosis) sequences were used as outgroups, respectively. Sequences from distantly related taxa are not generally applied as outgroups, as phylogenetic distance can lead to degraded alignments [43]. However, alignment degradation was not observed, which is consistent with similar reports using outgroups from distant taxa for CYP analyses [42].
\end{abstract}

\section{Functional analysis of $\mathrm{N}$-methyltransferase gene candidates}

Six gene candidates with greater than $40 \%$ sequence identity to one or more of four query sequences encoding known $N$-methyltransferases (NMTs) with established roles in BIA biosynthesis (Fig. 4; Additional file 6) were identified in the Glaucium flavum Illumina-based transcriptome. Coding sequences were amplified using gene-specific primers containing attB sites using Q5 HiFi DNA polymerase (New England Biolabs) and G. flavum 
root cDNA. Recombination reactions were carried out using BP and LR Clonase II (Thermo Scientific) to generate pDONR221-GfMMT entry plasmids and pHGWA-GfNMT expression plasmids. Heterologous protein expression was carried out at $16{ }^{\circ} \mathrm{C}$ using Escherichia coli ArcticExpress (Agilent Technologies) grown in Studier's autoinduction media (ZYP-5052) (Amresco, Solon, Ohio). Total soluble protein was extracted from each culture and the presence of His-tagged recombinant protein was verified by immunoblot procedure according to manufacturer's instructions (SuperSignal West Pico Chemiluminescent Substrate kit, Thermo Scientific). Five alkaloids (canadine, coclaurine, stylopine, reticuline, pavine) were screened in triplicate as potential substrates for G. flavum NMTs using a standardized assay under linear product formation conditions $(30 \mu \mathrm{g}$ total protein, $100 \mu \mathrm{M}$ alkaloid, $200 \mu \mathrm{M} S$-adenosyl methionine, $100 \mathrm{mM}$ sodium phosphate, $\mathrm{pH}$ 7). Total assay volume was $100 \mu \mathrm{L}$, and assays proceeded at $30{ }^{\circ} \mathrm{C}$ for either 5 or $30 \mathrm{~min}$, depending on the linear range pre-determined for each enzyme. Assays were analyzed by LC-MS/MS as previously described [9]. Most products were identified by comparison with retention times and CID spectra of authentic standards. $N, N$-Dimethylcoclaurine was identified by comparing the reaction product CID spectrum with previously reported data [8]. Product formation was monitored relative to empty vector controls. For each enzyme, activity was calculated relative to the assay in which the most substrate conversion was observed (i.e. the latter assay being set to $100 \%)$.

\section{Availability of supporting data}

All sequence data discussed in this paper have been deposited in the National Center for Biotechnology Information Sequence Read Archive (http://www.ncbi. nlm.nih.gov/sra) under the accession numbers listed in Additional files 3 and 4. All phylogenetic data are available in Dryad (http://dx.doi.org/10.5061/dryad.bh276).

\section{Additional files}

Additional file 1: Selected examples of BIA structural subgroups derived from the basic benzylisoquinoline subunit. (PDF $854 \mathrm{~kb}$ ) Additional file 2: Phylogenetic relationships among the Ranunculales as evidenced by molecular loci and morphological data. Adapted from [50]. (PDF $629 \mathrm{~kb}$ )

Additional file 3: Summary of results obtained using Roche-based deep sequencing platform. Predicted full-length CDS intersects between Roche-based and Illumina-based transcriptomes are indicated for comparison purposes (see Results and Discussion). (PDF $73 \mathrm{~kb}$ )

Additional file 4: Summary of results obtained using the Illuminabased deep sequencing platform. (PDF $80 \mathrm{~kb}$ )

Additional file 5: Summary of results obtained using the Illuminabased deep sequencing platform. For comparison purposes, both stem and root of Chelidonium majus were subjected to transcriptome analysis. (PDF $55 \mathrm{~kb}$ )
Additional file 6: FASTA file of all candidates, queries, and outgroups. (PDF $253 \mathrm{~kb}$ )

Additional file 7: Normalized expression analysis for gene candidates potentially involved in BIA biosynthesis in Papaveraceae (tribe: Fumariaceae) and Ranunculaceae species. Each candidate is labeled with respective species abbreviations (e.g. CCH, Corydalis chelanthifolia) and the type of enzyme potentially encoded by the gene (e.g. BBE, berberine bridge enzyme). Refer to Table 1 and Fig. 2 for species and enzyme/protein family abbreviations, respectively. Expression analysis was not performed for Mahonia aquifolium due to reduced numbers of full-length CDSs (see Results and Discussion).

Candidates present exclusively in Roche-based transcriptomes could not be assigned an FPKM value, and are marked with asterisks. (PDF $131 \mathrm{~kb}$ )

Additional file 8: Normalized expression analysis for gene candidates potentially involved in BIA biosynthesis in Menispermaceae species. Each candidate is labeled with respective species abbreviations (e.g. MCA, Menispermum canadense) and the type of enzyme potentially encoded by the gene (e.g. BBE, berberine bridge enzyme). Refer to Table 1 and Fig. 2 for species and enzyme/protein family abbreviations, respectively. Expression analysis was not performed for Cocculus trilobus due to reduced numbers of full-length CDSs (see Results and Discussion). Candidates present exclusively in Roche-based transcriptomes could not be assigned an FPKM value, and are marked with asterisks. (PDF $65 \mathrm{~kb}$ )

Additional file 9: Phylogenetic analysis of norcoclaurine synthase (NCS) gene candidates from twenty BIA-accumulating plant species. Red text denotes characterized genes or enzymes used as tBLASTn queries for transcriptome mining. Black text denotes uncharacterized gene candidates identified through mining ( $>40 \%$ identity to queries). Bootstrap values for each clade were based on 1000 iterations. Each candidate is labeled with respective species abbreviation (e.g. AME, Argemone mexicana; see Table 1) and candidate number (e.g. NCS1). Each query is labeled according to species (additional species: PSO, Papaver somniferum). The outgroup is Bet V1 allergen protein from Betula pendula (BPE). Amino acid sequences for candidates, queries, and outgroups are found in Additional file 6. (PDF $7248 \mathrm{~kb}$ )

Additional file 10: Phylogenetic analysis of berberine bridge enzyme (BBE) gene candidates from twenty BIA-accumulating plant species. Red text denotes characterized genes or enzymes used as tBLASTn queries for transcriptome mining. Black text denotes uncharacterized gene candidates identified through mining ( $>40 \%$ identity to queries). Species for which tBLASTn querying did not yield hits are not represented on the tree. Bootstrap values for each clade were based on 1000 iterations. Each candidate is labeled with

respective species abbreviation (e.g. AME, Argemone mexicana; see Table 1) and candidate number (e.g. BBE1) where more than one hit was identified. Each query is labeled according to species (additional species: PSO, Papaver somniferum; BST, Berberis stolonifera). The outgroup is tetrahydrocannabinolic acid synthase (THCAS) from Cannabis sativa (CSA). Amino acid sequences for candidates, queries, and outgroups are found in Additional file 6. (PDF $1851 \mathrm{~kb}$ )

Additional file 11: Phylogenetic analysis of FAD-dependent oxidase (FADOX) gene candidates from twenty BIA-accumulating plant species. Red text denotes characterized genes or enzymes used as tBLASTn queries for transcriptome mining. Black text denotes uncharacterized gene candidates identified through mining (>40 \% identity to queries). Species for which tBLASTn querying did not yield hits are not represented on the tree. Bootstrap values for each clade were based on 1000 iterations. Each candidate is labeled with respective species abbreviation (e.g. AME, Argemone mexicana; see Table 1). Each query is labeled according to species (PSO, Papaver somniferum; CJA, Coptis japonica; BWI, Berberis wilsoniae). The outgroup is tetrahydrocannabinolic acid synthase (THCAS) from Cannabis sativa (CSA). Amino acid sequences for candidates, queries, and outgroups are found in Additional file 6. (PDF $4025 \mathrm{~kb}$ )

Additional file 12: Phylogenetic analysis of 2-oxoglutarate/iron (II)-dependent dioxygenase (DIOX) gene candidates from twenty BIA-accumulating plant species. Red text denotes characterized genes or enzymes used as tBLASTn queries for transcriptome mining. Black text 
denotes uncharacterized gene candidates identified through mining (>40\% identity to queries). Bootstrap values for each clade were based on 1000 iterations. Each candidate is labeled with respective species abbreviation (e.g. AME, Argemone mexicana; see Table 1) and candidate number (e.g. DIOX1). The queries are derived from Papaver somniferum (PSO). The outgroup is anthocyanidin synthase from Arabidopsis thaliana (labeled ATHDIOX). Amino acid sequences for candidates, queries, and outgroups are found in Additional file 6. (PDF $20162 \mathrm{~kb}$ )

Additional file 13: Phylogenetic analysis of aldo-keto reductase gene candidates with homology to codeinone reductase (COR) from twenty BIA-accumulating plant species. Red text denotes the characterized COR1.3 from Papaver somniferum (PSO) used as a tBLASTn query for transcriptome mining. Black text denotes uncharacterized gene candidates identified through mining ( $>40 \%$ identity to query). Bootstrap values for each clade were based on 1000 iterations. Each candidate is labeled with respective species abbreviation (e.g. AME, Argemone mexicana; see Table 1) and candidate number (e.g. COR1). The outgroup is deoxymugineic acid synthase (DMAS) from Zea mays (ZMA). Amino acid sequences for candidates, queries, and outgroups are found in Additional file 6. (PDF $12593 \mathrm{~kb}$ )

Additional file 14: Phylogenetic analysis of short chain dehydrogenase/reductase gene candidates with homology to salutaridine reductase (SALR) from twenty BIA-accumulating plant species. Red text denotes the characterized SALR from Papaver somniferum (PSO) used as a tBLASTn query for transcriptome mining. Black text denotes uncharacterized gene candidates identified through mining ( $>40 \%$ identity to query). Species for which tBLASTn querying did not yield hits are not represented on the tree. Bootstrap values for each clade were based on 1000 iterations. Each candidate is labeled with respective species abbreviation (e.g. AME, Argemone mexicana; see Table 1) and candidate number (e.g. SALR1). The outgroup is porcine testicular carbonyl reductase (PTCR) from Sus scrofa (SSC). Amino acid sequences for candidates, queries, and outgroups are found in Additional file 6. (PDF $7467 \mathrm{~kb}$ )

\section{Additional file 15: Phylogenetic analysis of short chain} dehydrogenase/reductase gene candidates with homology to sanguinarine reductase (SANR) from twenty BIA-accumulating plant species. Red text denotes the characterized SANR1 from Eschschotzia californica (ECA) used as a tBLASTn query for transcriptome mining. Black text denotes uncharacterized gene candidates identified through mining (>40 \% identity to query). Bootstrap values for each clade were based on 1000 iterations. Each candidate is labeled with respective species abbreviation (e.g. AME, Argemone mexicana; see Table 1) and candidate number (e.g. SANR1). The outgroup is a SanR-like protein from Zea mays (ZMASDR). Amino acid sequences for candidates, queries, and outgroups are found in Additional file 6. (PDF $11309 \mathrm{~kb}$ )

\section{Additional file 16: Phylogenetic analysis of CYP80 gene} candidates from twenty BIA-accumulating plant species. Red text denotes characterized genes or enzymes used as tBLASTn queries for transcriptome mining. Black text denotes uncharacterized gene candidates identified through mining ( $>40 \%$ identity to queries). Bootstrap values for each clade were based on 1000 iterations. Each candidate is labeled with respective species abbreviation (e.g. AME, Argemone mexicana; see Table 1) and candidate number (e.g. CYP80-1). Each query is labeled according to species (additional species: HNI, Hyoscymus niger; CJA, Coptis japonica; BST, Berberis stolonifera; PSO, Papaver somniferum) with CYP80 subfamily and gene number indicated (e.g. CYP80A1, corytuberine synthase; see Fig. 1). The outgroup is CYP1B1 from Homo sapiens (HSA). Amino acid sequences for candidates, queries, and outgroups are found in Additional file 6. (PDF $13027 \mathrm{~kb}$ )

Additional file 17: Phylogenetic analysis of CYP82 gene candidates from twenty BIA-accumulating plant species. Red text denotes characterized genes or enzymes used as tBLASTn queries for transcriptome mining. Black text denotes uncharacterized gene candidates identified through mining (>40 \% identity to queries). Bootstrap values for each clade were based on 1000 iterations. Each candidate is labeled with respective species abbreviation (e.g. AME, Argemone mexicana; see Table 1) and candidate number (e.g. CYP82-1). Each query is labeled according to species (additional species: PSO, Papaver somniferum) with CYP82 subfamily and gene number indicated (e.g. CYP82N3, protopine hydroxylase; see Fig. 1). The outgroup is CYP82E4 from Nicotiana tabacum (NTA). Amino acid sequences for candidates, queries, and outgroups are found in Additional file 6. (PDF $7660 \mathrm{~kb}$ )

\section{Additional file 18: Phylogenetic analysis of $O$-methyltransferase} (OMT) gene candidates from twenty BIA-accumulating plant species. Red text denotes characterized genes or enzymes used as tBLASTn queries for transcriptome mining. Black text denotes uncharacterized gene candidates identified through mining ( $>40 \%$ identity to queries). Bootstrap values for each clade were based on 1000 iterations. Each candidate is labeled with respective species abbreviation (e.g. AME, Argemone mexicana; see Table 1) and candidate number (e.g. OMT1). Each query is labeled according to species (additional species: PSO, Papaver somniferum; CJA, Coptis japonica) and specific OMT function (SOMT, scoulerine O-methyltransferase; CbOMT, columbamine O-methyltransferase; N7OMT, norreticuline 7-O-methyltransferase; 7OMT; 6OMT; 4'OMT; see Fig. 1). The outgroup is isoflavone O-methyltransferase from Medicago sativa (MSA). Amino acid sequences for candidates, queries, and outgroups are found in Additional file 6. (PDF $13001 \mathrm{~kb}$ )

Additional file 19: Phylogenetic analysis of BAHD-type $O$-acetyltransferase (OAT; D'Aura 2006) gene candidates from twenty BIA-accumulating plant species. Red text denotes the characterized AT1 (1,13-dihydroxy-N-methylcanadine O-acetyltransferase; [6]) and SAT (salutaridine synthase) from Papaver somniferum (PSO) used as tBLASTn queries for transcriptome mining. Black text denotes uncharacterized gene candidates identified through mining (>30 \% identity to query). Bootstrap values for each clade were based on 1000 iterations. Each candidate is labeled with respective species abbreviation (e.g. AME, Argemone mexicana; see Table 1) and candidate number (e.g. AT1). The outgroup is vinorine synthase from Rauvolfia serpentina (RSEVS). Amino acid sequences for candidates, queries, and outgroups are found in Additional file 6. (PDF $5574 \mathrm{~kb}$ )

Additional file 20: Phylogenetic analysis of carboxyl esterase (CXE) gene candidates from twenty BIA-accumulating plant species. Red text denotes the characterized CXE1 (3-O-acetylpapaveroxine carboxylesterase; [6]) from Papaver somniferum (PSO) used as a tBLASTn query for transcriptome mining. Black text denotes uncharacterized gene candidates identified through mining ( $>30 \%$ identity to query). Bootstrap values for each clade were based on 1000 iterations. Each candidate is labeled with respective species abbreviation (e.g. AME, Argemone mexicana; see Table 1) and candidate number (e.g. CXE1). The outgroup is carboxylesterase 1 from Actinidia eriantha (AERCXE). Amino acid sequences for candidates, queries, and outgroups are found in Additional file 6. (PDF $6985 \mathrm{~kb}$ )

Additional file 21: Phylogenetic analysis of short chain dehydrogenase/reductase gene candidates with homology to noscapine synthase (NOS; [6]) from 20 BIA-accumulating plant species. Red text denotes the characterized NOS from Papaver somniferum (PSO) used as a tBLASTn query for transcriptome mining. Black text denotes uncharacterized gene candidates identified through mining ( $>40 \%$ identity to query). Bootstrap values for each clade were based on 1000 iterations. Each candidate is labeled with respective species abbreviation (e.g. AME, Argemone mexicana; see Table 1) and candidate number (e.g. NOS1). The outgroup is ben1-1D (BEN1) from Arabidopsis thaliana. Amino acid sequences for candidates, queries, and outgroups are found in Additional file 6. (PDF $8376 \mathrm{~kb}$ )

Additional file 22: Immunoblot analysis revealing the presence of His-tagged recombinant protein in soluble extracts of Escherichia coli strains expressing one of six $\mathrm{N}$-methyltransferase candidates (GFLNMT1-6) from Glaucium flavum. (PDF $203 \mathrm{~kb}$ )

\section{Abbreviations}

AKR: Aldo-keto reductase; BBE: Berberine bridge enzyme;

BIA: Benzylisoquinoline alkaloid; CDS: Coding sequence; COR: Codeinone reductase; CXE: Carboxylesterase; CYP: Cytochrome P450 monooxygenase; DIOX: 2-oxoglutarate/Fe $\mathrm{e}^{2+}$-dependent dioxygenase; FADX: FAD-dependent oxidoreductase, FPKM, Fragments per kilobase of exon model per million mapped reads; OAT: O-acetyltransferase; OMT: O-methyltransferase; 
NCS: Norcoclaurine synthase; NOS: Noscapine synthase; SalR: Salutaridine reductase; SanR: Sanguinarine reductase.

\section{Competing interests}

PJF has filed a patent application related to this work.

\section{Authors' contributions}

JMH interpreted the data and wrote the manuscript; JSM performed the NMT assays; EJL and IDP extracted RNA, coordinated the DNA sequencing and performed the initial database analyses; CDB, LC, XC and SCF performed the BLAST analyses and prepared the phylogenetic trees; YZ, JS and CWS conducted the bioinformatics analyses; PJF conceived of the study, sourced the plants, prepared the figures and edited the manuscript. All authors read and approved the final manuscript.

\section{Acknowledgments}

We are grateful to Stéphane Bailleul and Renée Gaudette from the Jardin Botanique de Montréal for invaluable assistance and access to plant collections. This work was funded through grants from Genome Canada, Genome Alberta and the Government of Alberta. CDB and SCF were recipients of Natural Sciences and Engineering Research Council of Canada graduate scholarships. SCF also received an Alberta Ingenuity Technology Futures graduate scholarship. PJF held the Canada Research Chair in Plant Metabolic Processes Biotechnology.

\section{Author details}

${ }^{1}$ Department of Biological Sciences, University of Calgary, Calgary, AB T2N 1N4, Canada. ${ }^{2}$ Department of Biochemistry and Molecular Biology, University of Calgary, Calgary, AB T2N 4N1, Canada. ${ }^{3}$ Current address: Département de Chimie, Biochimie et Physique, Université du Québec à Trois-Rivières, Trois-Rivières, QC G9A 5H7, Canada. ${ }^{4}$ Current address: Institute of Molecular Biotechnology, Graz University of Technology, Graz A-8010, Austria.

\section{Received: 3 April 2015 Accepted: 15 August 2015}

Published online: 18 September 2015

\section{References}

1. Beaudoin GAW, Facchini PJ. Isolation and characterization of a CDNA encoding (S)-cis-N-methylstylopine 14-hydroxylase from opium poppy, a key enzyme in sanguinarine biosynthesis. Biochem Biophys Res Commum. 2013;431:597-603.

2. Blaskó G, Gula DJ, Shamma M. The phthalideisoquinoline alkaloids. J Nat Prod. 1982;45:105-22.

3. Chen Y, McCarthy D, Robinson M, Smyth GK. edgeR: differential expression analysis of digital gene expression data. Bioconductor User's Guide. 2014;1-78.

4. Chevreux B, Pfisterer T, Drescher B, Driesel AJ, Müller WE, Wetter T, et al. Using the miraEST assembler for reliable and automated mRNA transcript assembly and SNP detection in sequenced ESTs. Genome Resource. 2004;14:1147-59.

5. D'Auria JC. Acyltransferases in plants: a good time to be BAHD. Curr Opin Plant Biol. 2006:9:331-40

6. Dang TT, Chen X, Facchini PJ. Acetylation serves as a protective group in noscapine biosynthesis in opium poppy. Nat Chem Biol. 2015;11:104-6.

7. Dang TT, Facchini PJ. CYP82Y1 is N-methylcanadine 1-hydroxylase, a key noscapine biosynthetic enzyme in opium poppy. J Biol Chem. 2014;289:2013-26.

8. Desgagné-Penix I, Facchini PJ. Systematic silencing of benzylisoquinoline alkaloid biosynthetic genes reveals the major route to papaverine in opium poppy. Plant J. 2012;72:331-44.

9. Farrow SC, Facchini PJ. Dioxygenases catalyze O-demethylation and O, O-demethylenation with widespread roles in benzylisoquinoline alkaloid metabolism in opium poppy. J Biol Chem. 2013;288:28997-9012.

10. Farrow SC, Hagel JM, Facchini PJ. Transcript and metabolite profiling in cell cultures of 18 plant species that produce benzylisoquinoline alkaloids. Phytochemistry. 2012;77:79-88.

11. Gaasterland T, Sensen CW. MAGPIE: automated genome interpretation. Trends Genet. 1996;12:76-8.

12. Gözler B, Gözler T, Shamma M. Egenine: a possible intermediate in phthalideisoquinoline biogenesis. Tetrahedron. 1983;39:577-80.

13. Guindon S, Gascuel O. A simple, fast and accurate algorithm to estimate large phylogenies by maximum likelihood. Syst Biol. 2003;52:696-704.
14. Guo Z, Cai R, Su H, Li Y. Alkaloids in processed rhizoma Corydalis and crude rhizoma Corydalis analyzed by GC-MS. J Anal Methods Chem. 2014;2014:1-6.

15. Hagel JM, Beaudoin GAW, Fossati E, Ekins A, Martin VJJ, Facchini PJ. Characterization of a flavoprotein oxidase from opium poppy catalyzing the final steps in sanguinarine and papaverine biosynthesis. J Biol Chem. 2012;287:42972-83.

16. Hagel JM, Facchini PJ. Dioxygenases catalyze the O-demethylation steps of morphine biosynthesis in opium poppy. Nat Chem Biol. 2010;6:273-5.

17. Hagel JM, Facchini PJ. Benzylisoquinoline alkaloid metabolism: a century of discovery and a brave new world. Plant Cell Physiol. 2013;54:647-72.

18. Hagel JM, Mandal, R, Han BS, Han J, Dinsmore DR, Borchers CH, et al. Metabolome analysis of 20 taxonomically related benzylisoquinoline alkaloid-producing plants. BMC Plant Biol. 2014. doi:10.1186/s12870-0150594-2.

19. Hawkins KM, Smolke CD. Production of benzylisoquinoline alkaloids in Saccharomyces cerevisiae. Nat Chem Biol. 2008;4:564-73.

20. Huang X, Madan A. CAP3: a DNA sequence assembly program. Genome Res. 1999;9:868-77.

21. Iranshahy M, Quinn RJ, Iranshahi M. Biologically active isoquinoline alkaloids with drug-like properties from the genus Corydalis. RSC Adv. 2014;4:15900-13.

22. Iwasa K, Takahashi T, Nishiyama Y, Moriyasu M, Sugiura M, Takeuchi A, et al. Online structural information of alkaloids and other constituents in crude extracts and cultured cells of Nandina domestica by combination of LC-MS/ MS, LC-NMR, and LC-CD analyses. J Nat Prod. 2008;71:1376-85.

23. Kavanagh $\mathrm{KL}$, Jörnvall H, Persson B, Oppermann U. The SDR superfamily: functional and structural diversity within a family of metabolic and regulatory enzymes. Cell Mol Life Sci. 2008;65:3895-906.

24. Küppers FJEM, Salemink CA, Bastart M, Paris M. Alkaloids of Papaver bracteatum: presence of codeine, neopine and alpinine. Phytochemistry. 1976;15:444-5.

25. Langmead B, Trapnell C, Pop M, Salzberg SL. Ultrafast and memory-efficient alignment of short DNA sequences to the human genome. Genome Biol. 2009; 10:R25.

26. Le PM, McCooeye M, Windust A. Application of UPLC-QTOF-MS in MS mode for the rapid and precise identification of alkaloids in goldenseal (Hydrastis canadensis). Anal Bioanal Chem. 2014;406:1739-49.

27. Lee EJ, Facchini PJ. Norcoclaurine synthase is a member of the pathogenesis-related 10/Bet v1 protein family. Plant Cell. 2010;22:3489-503.

28. Lee JW, Na D, Park JM, Lee J, Choi S, Lee SY. Systems metabolic engineering of microorganisms for natural and non-natural chemicals. Nat Chem Biol. 2012;8:536-46.

29. Li B, Dewey CN. RSEM: accurate transcript quantification from RNASeq data with or without a reference genome. BMC Bioinformatics. 2011;12:323.

30. Li W, Godzik A. Cd-hit: a fast program for clustering and comparing large sets of protein or nucleotide sequences. Bioinformatics. 2006;22:1658-9.

31. Li L, Stoeckert CJ, Roos DS. OrthoMCL: identification of ortholog groups for eukaryotic genomes. Genome Res. 2003;13:2178-89.

32. Li S, Tighe SW, Nicolet CM, Grove D, Levy S, Farmerie W, et al. Multiplatform assessment of transcriptome profiling using RNA-seq in the ABRF nextgeneration sequencing study. Nat Biotechnol. 2014;32:915-25.

33. Liscombe DK, Facchini PJ. Molecular cloning and characterization of tetrahydroprotoberberine cis- $\mathrm{N}$-methyltransferase, an enzyme involved in alkaloid biosynthesis in opium poppy. J Biol Chem. 2007;282:14741-51.

34. Liscombe DK, MacLeod BP, Loukanina N, Nandi OI, Facchini PJ. Evidence for the monophyletic evolution of benzylisoquinoline alkaloid biosynthesis in angiosperms. Phytochemistry. 2005;66:2501-20.

35. Liscombe DK, Ziegler J, Schmidt J, Ammer C, Facchini PJ. Targeted metabolite and transcript profiling for elucidating enzyme function: isolation of novel $\mathrm{N}$-methyltransferases from three benzylisoquinoline alkaloid-producing species. Plant J. 2009;60:729-43.

36. Margulies M, Egholm M, Altman WE, Attiya S, Bader JS, Bemben LA, et al. Genome sequencing in microfabricated high density picolitre reactors. Nature. 2005:437:376-80.

37. Martin JA, Wang Z. Next-generation transcriptome assembly. Nat Rev Genet. 2011;12:671-82.

38. Meisel L, Fonseca B, Gonzalez S, Baeza-Yates R, Cambiazo V, Campos R, et al. $A$ rapid and efficient method for purifying high quality total RNA from peaches (Prunus persica) for functional genomics analyses. Biol Res. 2005;38:83-8.

39. Mindnich RD, Penning TM. Aldo-keto reductase (AKR) superfamily: genomics and annotation. Hum Genomics. 2009;3:362-70. 
40. Nagarajan N, Pop M. Sequence assembly demystified. Nat Rev Genet. 2013;14:157-67.

41. Natajaran B, Paulsen BS. An ethnopharmacological study from Thane district, Maharashtra, India: traditional knowledge compared with modern biological science. Pharmaceut Biol. 2000;38:139-51.

42. Nelson DR. Plant cytochrome P450s from moss to poplar. Phytochem Rev. 2006:5:193-204.

43. Retief JD. Phylogenetic analysis using PHYLIP. In: Misener S, Krawetz SA, editors. Bioinformatics: Methods and Protocols. New York: Humana Press; 1999. p. 243-58.

44. Sato F. Characterization of plant functions using cultured plant cells, and biotechnological applications. Biosci Biotechnol Biochem. 2013;77:1-9.

45. Semwal DK, Semwal RB, Vermaak I, Viljoen A. From arrow poison to herbal medicine - the ethnobotanical, phytochemical and pharmacological significance of Cissampelos (Menispermaceae). J Ethnopharmacol. 2014;155:1011-28.

46. SEQC/MAQC-III Consortium. A comprehensive assessment of RNA-seq accuracy, reproducibility and information content by the Sequencing Quality Control Consortium. Nat Biotechnol. 2014;32:903-14.

47. Shamma M, Salgar SS. Pallidine and corydine from Thalictrum dioicum. Phytochemistry. 1973;12:1505-6.

48. Thodey K, Galanie S, Smolke CD. A microbial biomanufacturing platform for natural and semisynthetic opioids. Nat Chem Biol. 2014;10:837-44.

49. Wang C, Gong B, Bushel PR, Thierry-Mieg J, Thierry-Mieg D, Xue J, et al. The concordance between RNA-seq and microarray data depends on chemical treatment and transcript abundance. Nat Biotechnol. 2014;32:926-32.

50. Wang W, Lu A-M, Ren Y, Endress ME, Chen Z-D. Phylogeny and classification of Ranunculales: evidence from four molecular loci and morphological data. Perpect Plant Ecol. 2009;11:81-110.

51. Winzer T, Gazda V, He Z, Kaminski F, Kern M, Larson TR, et al. A Papaver somniferum 10-gene cluster for synthesis of the anticancer alkaloid noscapine. Science. 2012;336:1704-8.

52. Wu C, Yan R, Zhang R, Bai F, Yang Y, Wu Z, et al. Comparative pharmacokinetics and bioavailability of four alkaloids in different formulations from Corydalis decumbens. J Ethnopharmacol. 2013;149:55-61.

53. Xiao M, Zhang Y, Chen X, Lee E-J, Barber CJS, Chakrabarty R, et al. Trancriptome analysis based on next-generation sequencing of non-model plants producing specialized metabolites of biotechnological interest. J Biotechnol. 2013:166:122-34.

54. Yue W, Ming Q-L, Lin B, Rahman K, Zheng C-J, Han T, et al. Medicinal plant cell suspension cultures: pharmaceutical applications and high-yielding strategies for the desired secondary metabolites. Crit Rev Biotechnol. 2014. Early Online ed: 1-18.

55. Zerbino DR, Birney E. Velvet: algorithms for de novo short read assembly using de Bruijn graphs. Genome Res. 2008;18:821-9.

56. Zhang Z, Schwartz S, Wagner L, Miller W. A greedy algorithm for aligning DNA sequences. J Comput Biol. 2000;7:203-14

\section{Submit your next manuscript to BioMed Central and take full advantage of:}

- Convenient online submission

- Thorough peer review

- No space constraints or color figure charges

- Immediate publication on acceptance

- Inclusion in PubMed, CAS, Scopus and Google Scholar

- Research which is freely available for redistribution 